\title{
Population Genetics for 15 STR loci of Liaoning Han in Northeastern China
}

\author{
Yongji Zhang* \\ Department of Forensic Medicine, Yanbian University College of Medicine, Yanji City, Jilin Province, China
}

\begin{abstract}
Allele frequencies for the 15 autosomal STR loci D3S1358, TH01, D21S11, D18S51. Penta E, D5S818, D13S317, D7S820, D16S539, CSF1PO, Penta D, vWA, D8S1179, TPOX, and FGA (PowerPlex ${ }^{\circledR} 16$ system $^{\mathrm{Tm}}$, Promega corporation)were determined in a population sample of 538 unrelated individuals from northeastern China Han (Liaoning).
\end{abstract}

Keywords: STR; Allele frequencies; Northeastern China Han; Pow$\operatorname{erPlex}^{\circledR} 16$ system $^{\text {sm }}$

\section{Population}

Boold samples were collected from 538 unrelated individuals from the northeastern China Han(Liaoning).

\section{Extraction}

DNA was extracted from whole blood using the Chelex-100 extraction method [1].

\section{PCR}

PCR amplification was performed using $1.0 \mathrm{ng}$ genomic DNA template according to manufacturer's protocol (PowerPlex 16 system", Promega Corporation) [2].

\section{Typing}

PCR products were separated on an ABI Prism 3100 Genetic Analyzer (Applied Biosystems) using the recommended protocol. The results were analyzed by Data Collection (Version 1.1), GeneScan (Version 3.7), and Genotyper (Version 3.6) softwares (Applied Biosystems)

\section{Analysis of Data}

Allele frequencies and summary statistics were calculated with "PowerStats V 12" p rogram (Promega home page:http://www. promega.com/geneticidtools/). Estimations of Hardy-Weinberg equilibrium according to Guo and Thompson [3]. To compare our data to those from other group samples, Arlequin 2.0 was used to calculate pairwise diffrencese for each locus [4].

\section{Quality control}

Laboratory Internal Control Standards and Kit controls.

\section{Access to the data}

Via electronic mail from communicating author: zhangyj@ybu. edu.cn.

\section{Results}

Allele frequencies for each locus are presented in Table 1. Forensic statistics for the 15 loci are summarized in Table 2.

\section{Other Remarks}

No significant deviation from Hardy-Weinberg expectations $(p>$ 0.05 ) for 15 STR loci. A comparative analysis between our data and previously published data for the same loci has been done using the statistical method of pairwise differentiation test. The comparative results showed that northeastern China (Liaoning) Han population has significant differences from Caucasian at 10 locus (except D3S1358, D18S51, Penta D, D8S1179 and FGA) [5], African at 13 locus (except D3S1358 and D16S539) [6], respectively. And there were no significant difference between the studied population and Beijing Han [7], Sichuan Han (except D18S51) [8], Hongkong Han (except TH01 and D18S51) [9], Southeastern Han [10], Northwestern Han (except D18S51) [11], South Korean (except TH01) [12], and Japanese (except TH01) [13, 14]. The combined MP (Matching Probability) and PE (power of exclusion) for 15 STR loci were $5.76 \times 10^{-18}$ and 0.999998 , respectively.

This paper follows the guidelines for publication of population data requested by the journal [15].

*Corresponding author: Yongji Zhang, Department of Forensic Medicine, Yanbian University College of Medicine, 977 gongyuan road, 133002 Yanji City, Jilin Province, P.R. China, E-mail: zhangyi@ybu.edu.cn

Received March 10, 2011; Accepted April 07, 2011; Published April 11, 2011

Citation: Zhang Y (2011) Population Genetics for 15 STR loci of Liaoning Han in Northeastern China. J Forensic Res 2:123. doi:10.4172/2157-7145.1000123

Copyright: () 2011 Zhang Y. This is an open-access article distributed under the terms of the Creative Commons Attribution License, which permits unrestricted use, distribution, and reproduction in any medium, provided the original author and source are credited. 
Citation: Zhang Y (2011) Population Genetics for 15 STR loci of Liaoning Han in Northeastern China. J Forensic Res 2:123. doi:10.4172/21577145.1000123

Page 2 of 3

\begin{tabular}{|c|c|c|c|c|c|c|c|c|c|c|c|c|c|c|c|}
\hline & D3S1358 & TH01 & D21S11 & D18S51 & Penta E & D5S818 & D13S317 & D7S820 & D16S539 & CSF1PO & Penta D & vWA & D8S1179 & TPOX & FGA \\
\hline Allele & $n=538$ & $n=538$ & $n=538$ & $n=538$ & $n=538$ & $n=538$ & $n=538$ & $n=538$ & $n=538$ & $n=538$ & $n=538$ & $n=538$ & $n=538$ & $n=538$ & $n=538$ \\
\hline 5 & & & & & 0.0428 & & & & & & & & & & \\
\hline 6 & & 0.1050 & & & & & & & & & 0.0028 & & & & \\
\hline 7 & & 0.2416 & & & 0.0019 & 0.0093 & 0.0065 & & & 0.0019 & 0.0056 & & & 0.0009 & \\
\hline 8 & & 0.0530 & & & 0.0037 & 0.0009 & 0.2630 & 0.1292 & 0.0065 & 0.0009 & 0.0436 & & 0.0009 & 0.5325 & \\
\hline 9 & & 0.5437 & & & 0.0084 & 0.0594 & 0.1227 & 0.0799 & 0.2890 & 0.0604 & 0.2931 & & 0.0009 & 0.1125 & \\
\hline 9.3 & & 0.0325 & & & & & & & & & & & & & \\
\hline 10 & & 0.0214 & & 0.0019 & 0.0502 & 0.2189 & 0.1543 & 0.1599 & 0.1171 & 0.2128 & 0.1252 & & 0.0836 & 0.0251 & \\
\hline 11 & & 0.0028 & & 0.0037 & 0.1199 & 0.3219 & 0.2388 & 0.3522 & 0.2574 & 0.2342 & 0.1753 & & 0.0846 & 0.3011 & \\
\hline 12 & & & & 0.0381 & 0.0948 & 0.2347 & 0.1599 & 0.2435 & 0.2100 & 0.3931 & 0.1670 & & 0.1375 & 0.0270 & \\
\hline 13 & & & & 0.1896 & 0.0455 & 0.1391 & 0.0372 & 0.0316 & 0.1041 & 0.0855 & 0.1494 & & 0.2184 & 0.0009 & \\
\hline 14 & 0.0417 & & & 0.2165 & 0.0781 & 0.0102 & 0.0177 & 0.0037 & 0.0149 & 0.0102 & 0.0306 & 0.2649 & 0.1868 & & \\
\hline 15 & 0.3813 & & & 0.1719 & 0.1152 & 0.0037 & & & 0.0009 & 0.0009 & 0.0056 & 0.0344 & 0.1812 & & \\
\hline 16 & 0.3284 & & & 0.1301 & 0.0976 & 0.0009 & & & & & 0.0009 & 0.1691 & 0.0883 & & \\
\hline 17 & 0.1837 & & & 0.0641 & 0.0939 & & & & & & & 0.2305 & 0.0139 & & 0.0009 \\
\hline 18 & 0.0622 & & & 0.0595 & 0.0818 & 0.0009 & & & & & 0.0009 & 0.1989 & 0.0037 & & 0.0270 \\
\hline 19 & 0.0028 & & & 0.0362 & 0.0586 & & & & & & & 0.0836 & & & 0.0465 \\
\hline 20 & & & & 0.0279 & 0.0586 & & & & & & & 0.0158 & & & 0.0437 \\
\hline 20.2 & & & & & & & & & & & & & & & 0.0019 \\
\hline 21 & & & & 0.0279 & 0.0242 & & & & & & & 0.0028 & & & 0.1059 \\
\hline 21.2 & & & & & & & & & & & & & & & 0.0028 \\
\hline 22 & & & & 0.0186 & 0.0112 & & & & & & & & & & 0.1320 \\
\hline 22.2 & & & & & & & & & & & & & & & 0.0084 \\
\hline 22.3 & & & & & & & & & & & & & & & 0.0019 \\
\hline 23 & & & & 0.0093 & 0.0084 & & & & & & & & & & 0.2212 \\
\hline 23.2 & & & & & & & & & & & & & & & 0.0065 \\
\hline 24 & & & & 0.0046 & 0.0019 & & & & & & & & & & 0.2138 \\
\hline 24.2 & & & & & & & & & & & & & & & 0.0056 \\
\hline 25 & & & & & 0.0037 & & & & & & & & & & 0.0967 \\
\hline 25.2 & & & & & & & & & & & & & & & 0.0037 \\
\hline 26 & & & & & & & & & & & & & & & 0.0576 \\
\hline 26.2 & & & & & & & & & & & & & & & 0.0019 \\
\hline 27 & & & & & & & & & & & & & & & 0.0186 \\
\hline 28 & & & 0.0409 & & & & & & & & & & & & 0.0037 \\
\hline 28.2 & & & 0.0046 & & & & & & & & & & & & \\
\hline 29 & & & 0.2463 & & & & & & & & & & & & \\
\hline 29.2 & & & 0.0009 & & & & & & & & & & & & \\
\hline 30 & & & 0.3132 & & & & & & & & & & & & \\
\hline 30.2 & & & 0.0130 & & & & & & & & & & & & \\
\hline 30.3 & & & 0.0056 & & & & & & & & & & & & \\
\hline 31 & & & 0.1022 & & & & & & & & & & & & \\
\hline 31.2 & & & 0.0595 & & & & & & & & & & & & \\
\hline 32 & & & 0.0297 & & & & & & & & & & & & \\
\hline 32.2 & & & 0.1264 & & & & & & & & & & & & \\
\hline 33 & & & 0.0084 & & & & & & & & & & & & \\
\hline 33.2 & & & 0.0437 & & & & & & & & & & & & \\
\hline 34 & & & 0.0028 & & & & & & & & & & & & \\
\hline 34.2 & & & 0.0009 & & & & & & & & & & & & \\
\hline 35 & & & 0.0009 & & & & & & & & & & & & \\
\hline 35.2 & & & 0.0009 & & & & & & & & & & & & \\
\hline
\end{tabular}

Table 1: STR allele frequencies of 538 individuals from northeastern China(Liao Ning) Han. 
Citation: Zhang Y (2011) Population Genetics for 15 STR loci of Liaoning Han in Northeastern China. J Forensic Res 2:123. doi:10.4172/21577145.1000123

Page 3 of 3

\begin{tabular}{|c|c|c|c|c|c|c|c|c|c|c|c|c|c|c|c|}
\hline & D3S1358 & TH01 & D21S11 & D18S51 & Penta E & D5S818 & D13S317 & D7S820 & D16S539 & CSF1PO & Penta D & vWA & D8S1179 & TPOX & FGA \\
\hline $\mathrm{He}$ & 0.7075 & 0.6307 & 0.8065 & 0.8582 & 0.9199 & 0.7703 & 0.8076 & 0.7670 & 0.7813 & 0.7342 & 0.8146 & 0.8001 & 0.8435 & 0.6117 & 0.8588 \\
\hline $\mathrm{Ho}$ & 0.6902 & 0.6450 & 0.7881 & 0.8662 & 0.9349 & 0.7774 & 0.8011 & 0.7602 & 0.7770 & 0.7435 & 0.7885 & 0.7714 & 0.8383 & 0.6059 & 0.8606 \\
\hline PIC & 0.6558 & 0.5852 & 0.7813 & 0.8428 & 0.9121 & 0.7340 & 0.7801 & 0.7332 & 0.7470 & 0.6926 & 0.7900 & 0.7703 & 0.8241 & 0.5499 & 0.8438 \\
\hline PE & 0.4132 & 0.3484 & 0.5772 & 0.7270 & 0.8672 & 0.5577 & 0.6011 & 0.5274 & 0.5570 & 0.4987 & 0.5779 & 0.5470 & 0.6719 & 0.2981 & 0.7159 \\
\hline PD & 0.8657 & 0.8229 & 0.9392 & 0.9632 & 0.9852 & 0.9081 & 0.9348 & 0.9118 & 0.9126 & 0.8868 & 0.9400 & 0.9311 & 0.9556 & 0.7912 & 0.9636 \\
\hline$P$ & 0.6977 & 0.7612 & 0.2924 & 0.7479 & 0.0987 & 0.7173 & 0.7893 & 0.9609 & 0.1495 & 0.3675 & 0.1520 & 0.9558 & 0.1595 & 0.9817 & 0.2945 \\
\hline
\end{tabular}

He: Expected heterozygotes, Ho: Observed heterozygotes, PIC: Polymorphism information content., PE: Power of exclusion, PD: power of discrimination, p: probability values of exact tests for Hardy-Weinberg equilibrium.

Table 2: Statistical parameters of the 15 STR loci for forensic interest in northeastern China(Liao Ning) Han.

\section{References}

1. Walsh PS, Metzger DA, Higuchi R (1991) Chelex 100 as a medium for simple extraction of DNA for PCR-based typing from forensic material. Biotechniques 10: 506-513.

2. PowerPlex ${ }^{\circledR} 16$ system $^{\text {Tm }}$ Manual, Promega Corporation, USA.

3. Guo SW, Thompson EA (1992) Performing the exact test of Hardy-Weinberg proportion for multiple alleles. Biometrics 48: 361-372.

4. Schneider S, Roessli D, Excoffier L (2000) Arlequin Ver. 2.000: A Software for Population Genetics Data Analysis. Genetics and Biometry Laboratory, University of Geneva, Switzerland.

5. Figueiredo MS, Fernandez-Rosado F, Lorente JA, Budowle B (2004) Brazilian Caucasian population data for 15 STR loci (PowerPlex 16 kit). J Forensic Sci 49: 167-169.

6. Beleza S, Alves C, Reis F, Amorim A, Carracedo A, et al. (2004) 17 STR data (AmpF/STR Identifiler and Powerples 16 System) from Cabinda (Angola), Forensic Sci Int 141: 193-196.

7. Liu YC, Huo ZY, Tang H, Wang J, Jiao ZP, et al. (2003) Frequency data for 15 STR loci and forensic use in a Beijing-Han population. Int Congress Ser. 1239: $267-270$.

8. Zhang H, Li Y, Jiang J, Zhang J, Wu J, et al. (2007) Analysis of 15 STR loci in Chinese population from Sichuan in West China. Forensic Sci Int 171: 222-225.
9. Law MY, To KY, Ho SH, Pang BC, Wong LM, et al. (2002) STR data for the PowerPlex ${ }^{\text {Tw }} 16$ loci for the Chinese population in Hong Kong. Forensic Sci Int 129: 64-67.

10. Hu SP, Wu DQ, Xu XH, Liu JW, Li B (2005) Genetic profile of 15 STR loci in the Min Nan population in Southeast China. Forensic Sci Int 152: 263-265.

11. Wang ZY, Yu RJ, Wang F, Li XS, Jin TB (2005) Genetic polymorphisms of 15 STR loci in Han population from Shaanxi (NW China). Forensic Sci Int 147: 89-91.

12. Kim YL, Hwang JY, Kim YJ, Lee S, Chung NG, et al. (2003) Allele frequencies of 15 STR loci using AmpF/STR Identifiler kit in a Korean population. Forensic Sci Int 136: 92-95.

13. Tie J, Wang X, Oxida S (2006) Genetic polymorphisms of 15 STR loci in a Japanese population. J Forensic Sci 51: 188-189.

14. Hara M, Yamamoto Y, Takada A, Saito K, Kido A, et al. (2004) Population data for 15 STR loci D3S1358, TH01, D21S11, D18S51, Penta E, D5S818, D13S317, D7S820, D16S539, CSF1PO, Penat D, vWA, D8S1179, TPOX and FGA in Japanese. Int Congress Ser 1261: 204-206.

15. Lincoln P, Carracedo A (2000) Publication of population data of human polymorphisms. Forensic Sci Int 110: 3-5. 